\title{
Magnetic resonance neurography findings in three critically ill COVID-19 patients with new onset of extremity peripheral neuropathy
}

\author{
Imran M. Omar ${ }^{A, B, D, E, F}$, Ankur Garg ${ }^{D, E, F}$ \\ Department of Radiology, Northwestern University Feinberg School of Medicine, United States
}

\begin{abstract}
Purpose: Coronavirus-19 (COVID-19) is most commonly associated with respiratory syndromes, although patients are presenting more frequently with neurological symptoms. When they occur, neurological conditions most commonly involve the central nervous system, and peripheral nervous system effects, particularly in the extremities, have been less commonly described. The mechanisms of peripheral neuropathy in critically ill patients with COVID-19 are likely to be multifactorial, and extremity peripheral nerve imaging in these cases has not been well described.

Case presentation: In this case series, we describe the magnetic resonance neurography (MRN) findings in 3 critically ill patients who presented with new onset of peripheral neuropathies in the extremities, and we discuss possible common mechanisms of nerve injury, including the role of position-related nerve injury.

Conclusions: MRN can be useful in identifying and localizing peripheral nerve abnormalities in the extremities of COVID-19 patients, and patients who are placed in the prone position during ventilation may be more susceptible to these injuries.
\end{abstract}

Key words: MRI, prone position, neurography, COVID-19, peripheral nerve, critical-illness.

\section{Introduction}

Coronavirus-19 (COVID-19), which is caused by severe acute respiratory syndrome coronavirus 2 (SARS-Cov-2), has produced more than 54 million infections and 1.3 million deaths worldwide as of mid-November 2020 [1]. It has primarily been associated with a variety of respiratory syndromes, and the effect of COVID-19 on the nervous system, particularly the peripheral nervous system (PNS), is less well reported. These can be divided into conditions related directly to viral nerve invasion, postviral neuropathies, and treatment-related neuropathies. The earliest effects reported in the PNS involved the head and neck and spinal nerves, and they include anosmia or ageusia [2]. Peripheral neuropathies involving the extremities in patients with COVID-19 are probably due to several causes and have rarely been reported [3]. This may be because many patients who develop COVID-19-related peripheral neuropathies have other severe respiratory, thrombotic, or inflammatory conditions, the management of which supersedes evaluation of non-life-threatening conditions. Furthermore, these patients are often sedated or neurologically impaired and may be unable to provide an appropriate history or physical examination suggesting peripheral neuropathy. Finally, appropriate clinical assessment and imaging studies for nonurgent indications may be delayed until the patient is deemed no longer contagious. As a result, COVID-19 patients with new peripheral neuropathies may not be referred for diagnostic testing, such as diagnostic imaging or electrodiagnostic studies, until later in the course of their disease.

Magnetic resonance imaging (MRI) findings in COVID-19 patients with extremity peripheral neuropathies have not been well-reported. In this case series, we

Correspondence address:

Imran M. Omar, Department of Radiology, Northwestern University Feinberg School of Medicine, United States, e-mail: iomar@nm.org

Authors' contribution:

A Study design - B Data collection - C Statistical analysis · D Data interpretation - E Manuscript preparation · F Literature search · G Funds collection 
report the magnetic resonance neurography (MRN) imaging findings in 3 cases of critically ill COVID-19 patients who developed extremity peripheral neuropathies during the courses of their hospitalizations between July and October 2020 and discuss some of the common clinical features that may put these patients at risk of developing these conditions.

\section{Case presentations}

\section{Case 1}

Our earliest patient is a 21-year-old, previously healthy, Hispanic male with a BMI of $31.1 \mathrm{~kg} / \mathrm{m}^{2}$, who was admitted to our intensive care unit (ICU) for multifocal COVID-19 pneumonia and acute respiratory distress syndrome (ARDS) following a 5-day history of dyspnoea, cough, fevers, and watery diarrhoea. The patient was sedated and intubated, and given prednisone, Plaquenil, and a number of antibiotics, including azithromycin, ceftriaxone, vancomycin, meropenem and cefepime, to treat a superimposed ventilator-acquired pneumonia. While intubated, he was given a trial of prone positioning to improve his oxygenation.

Upon extubation, the patient noticed bilateral upper extremity weakness, including a right wrist drop and left shoulder sharp pain, weakness, and numbness. The patient's physical examination and history revealed a right radial nerve palsy and left shoulder weakness, particularly with abduction. MRI of the brain and cervical spine did not reveal a central cause for these symptoms, and lumbar puncture performed to assess for signs of encephalitis did not demonstrate any signs of infection or elevated cerebrospinal fluid protein concentration. The patient's serum creatinine kinase (CK) levels briefly and mildly peaked 2 days after admission at 287 units/liter (U/l) (normal at our institution is $0-223 \mathrm{U} / \mathrm{l})$.
Due to more pressing respiratory issues, electrodiagnostic assessment and left upper extremity imaging occurred nearly 4 weeks after the patient first noted his symptoms. Electrodiagnostic studies of each upper extremity confirmed a distal right radial mononeuropathy and moderate left shoulder infraspinatus and deltoid muscle dysfunction, suggesting a subacute C5 radiculopathy or brachial plexopathy involving the upper trunk. Left shoulder MRI showed diffuse infraspinatus greater than supraspinatus muscle oedema, as well as anterior and lateral deltoid head diffuse muscle oedema. The constellation of findings suggested brachial plexopathy, possibly related to prior traction injury, or post-viral Parsonage-Turner syndrome.

Subsequently, a left brachial plexus MRN was performed to better assess the regional peripheral nerves (Figure 1), which showed extensive left C5 and C6 nerve root oedema extending to the superior trunk, anterior and posterior divisions, and the posterior cord. The posterior cord gives rise to the axillary and suprascapular nerves, which were also oedematous and innervated the involved muscles in the shoulder. Although both the C5 and C6 nerves appeared oedematous, only the $\mathrm{C} 5$ nerve root enhanced following intravenous contrast administration, which was thought to possibly reflect a higher-grade injury.

Since his discharge, the patient has improved after performing physical therapy but exhibits residual shoulder weakness.

\section{Case 2}

Our next patient is a 61-year-old Hispanic male with a BMI of $31.1 \mathrm{~kg} / \mathrm{m}^{2}$, who developed COVID-19 pneumonia and ARDS after 5 days of fever, chills, dizziness, pleuritic chest pain, abdominal pain, and ageusia. The patient was given remdesivir and steroids and transferred to our ICU after 7 days, where he was sedated with neuro-
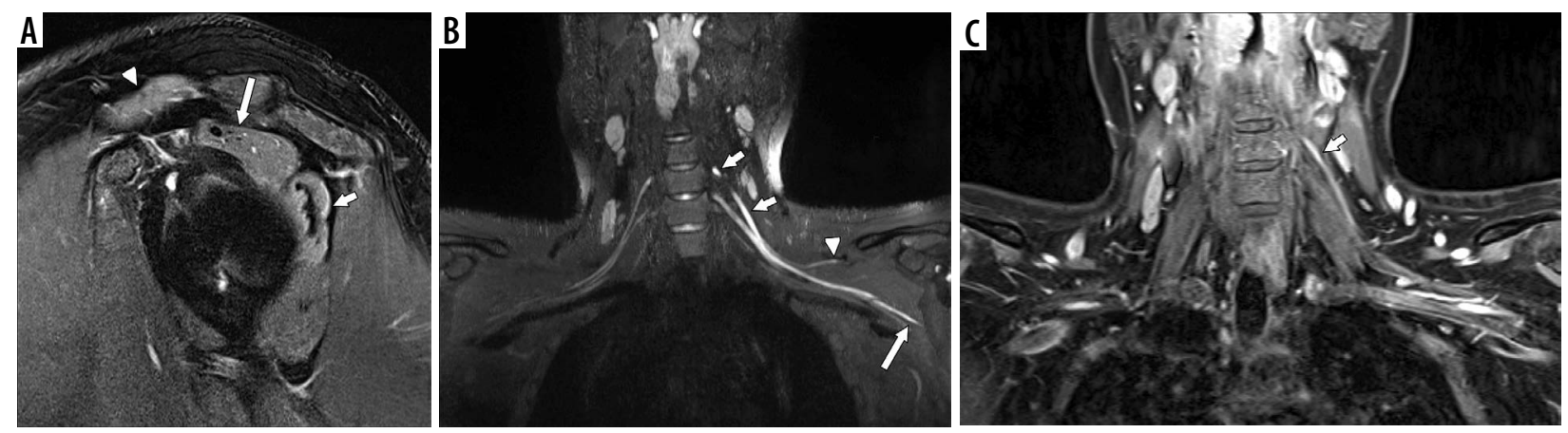

Figure 1.3T magnetic resonance neurography in a 21-year-old male with COVID-19-related acute respiratory distress syndrome and right radial nerve palsy and left shoulder weakness noted after extubation. A) Sagittal oblique T2W FS MR image of the shoulder demonstrates diffuse muscle oedema of the infraspinatus (shorter arrow), supraspinatus (longer arrow), and anterior deltoid muscles (arrowhead). B) Coronal maximal intensity projection reformatted image from a thin-section, large field-of-view coronal 3D STIR SPACE sequence of the brachial plexus shows extensive, asymmetric left brachial plexus oedema of the $C 5$ nerve root extending into the superior trunk (shorter arrows), the (6 nerve root, the suprascapular nerve (arrowhead), and the axillary nerve (longer arrow). The patient had motor deficits of the anterior and lateral deltoid muscles and the supraspinatus and infraspinatus muscles, which corresponded to axillary and suprascapular nerve deficits. C) Coronal large field-of-view T1W FS postcontrast magnetic resonance image of the brachial plexus depicts enhancement of the $C 5$ nerve root only as it extends to the superior trunk (arrow). This corresponded to the findings from electrodiagnostic studies 
muscular blockade, intubated, and given nitrous oxide to improve oxygenation. Due to persistent hypoxaemia, he underwent a few cycles of prone positioning while intubated. Following extubation, the patient noted new left upper extremity weakness, particularly when trying to flex the elbow.

His neurological examination revealed 1/5 strength with elbow flexion and shoulder adduction, 4/5 strength with elbow extension, and 3/5 strength with wrist flexion and extension. His sensation and reflexes were intact. Electrodiagnostic studies suggested an upper trunk brachial plexopathy, active deltoid muscle denervation, and mild ulnar sensory neuropathy. Serum CK levels were elevated for 2 days, peaking 12 days after admission at $710 \mathrm{U} / \mathrm{l}$.

Cervical spine MRI did not reveal clear findings of impingement that would explain the patient's deficits, and left brachial plexus MRN was performed (Figure 2). Large field-of-view coronal STIR imaging demonstrated symmetric muscle and fascial oedema and enhancement of the shoulder girdles, particularly the supraspinatus, infraspinatus, and teres minor muscles. The brachial plexus nerves on each side were unremarkable. However, the left musculocutaneous nerve and biceps brachii muscle were diffusely oedematous and enhancing. Given the patient's symptoms and MRI findings, the rotator cuff muscle oedema was thought to relate to critical-illness myopathy, whereas the musculocutaneous nerve and biceps brachii findings were more likely to be related to position-related neuropathy.
Following discharge, the patient's left upper extremity symptoms have improved with physical therapy, although there is moderate residual weakness on elbow flexion.

\section{Case 3}

Our most recent patient is a 37-year-old Hispanic, morbidly obese male with a BMI of $47.9 \mathrm{~kg} / \mathrm{m}^{2}$, asthma, and type II diabetes mellitus. The patient experienced 10 days of progressive cough, dyspnoea, and chest pain with exertion, and was admitted to our ICU with COVID-19 ARDS, along with diabetic ketoacidosis, acute renal insufficiency, and superimposed streptococcal pneumonia. In the ICU, the patient was given remdesivir, sedated, placed on a ventilator, and given one episode of prone positioning due to worsening respiratory failure.

Following extubation, the patient immediately noted numbness, throbbing, pain, and swelling of his right foot, which were exacerbated when lying supine and improved with walking. On physical examination, the patient had $0 / 5$ strength on foot dorsiflexion, plantarflexion, inversion, and eversion, as well as 0/5 extensor hallucis longus muscle strength. Knee flexion demonstrated $4 / 5$ strength, and he had no discernible Achilles reflex. The patient also had decreased sensation over the lateral calf and entire foot. The differential diagnosis included sensorimotor L5-S1 radiculopathy, post-infectious peripheral neuropathy or lumbar plexopathy that was possibly related to compression experienced while being in the prone position.
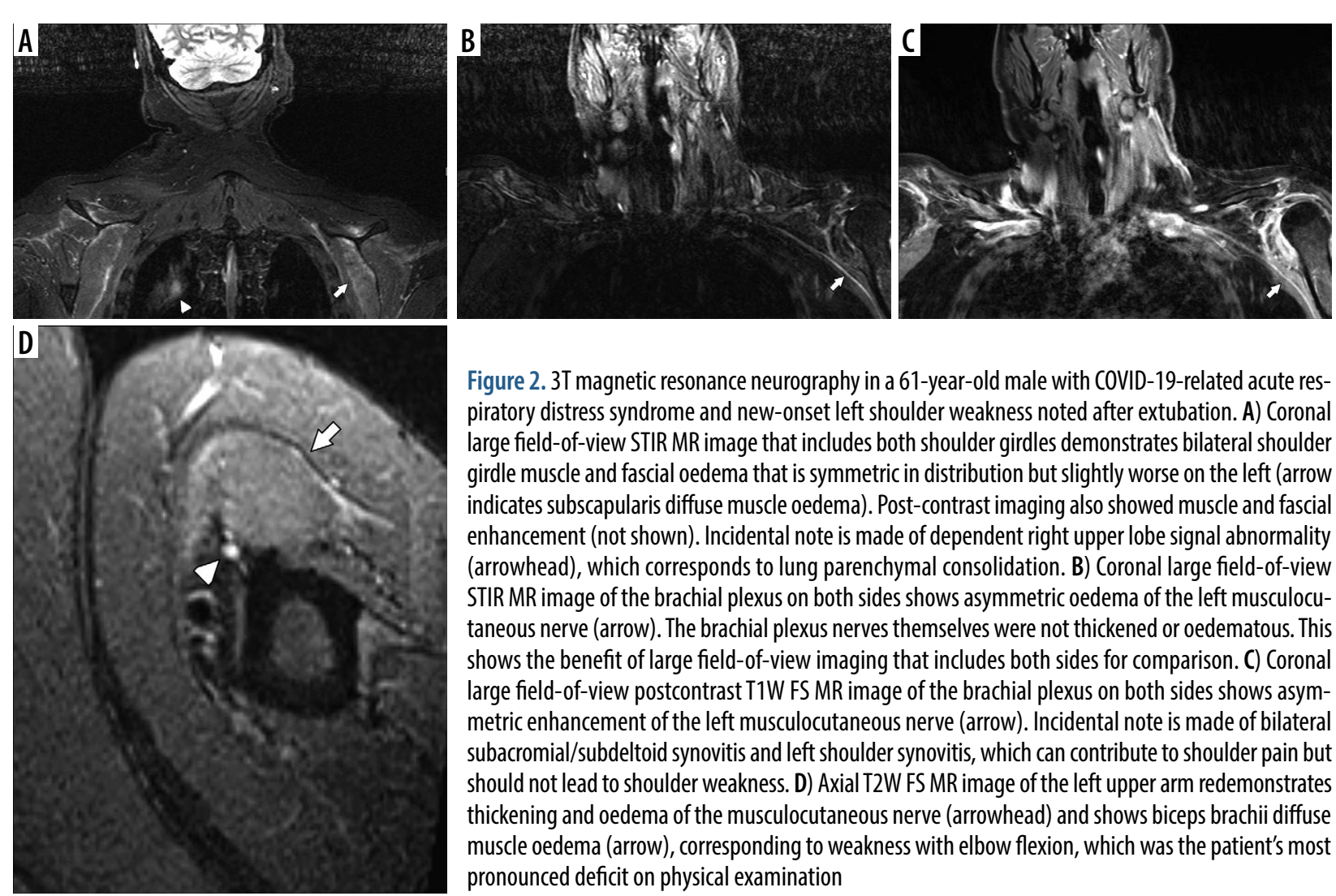

Figure 2.3T magnetic resonance neurography in a 61-year-old male with COVID-19-related acute respiratory distress syndrome and new-onset left shoulder weakness noted after extubation. A) Coronal large field-of-view STIR MR image that includes both shoulder girdles demonstrates bilateral shoulder girdle muscle and fascial oedema that is symmetric in distribution but slightly worse on the left (arrow indicates subscapularis diffuse muscle oedema). Post-contrast imaging also showed muscle and fascial enhancement (not shown). Incidental note is made of dependent right upper lobe signal abnormality (arrowhead), which corresponds to lung parenchymal consolidation. B) Coronal large field-of-view STIR MR image of the brachial plexus on both sides shows asymmetric oedema of the left musculocutaneous nerve (arrow). The brachial plexus nerves themselves were not thickened or oedematous. This shows the benefit of large field-of-view imaging that includes both sides for comparison. C) Coronal large field-of-view postcontrast T1W FS MR image of the brachial plexus on both sides shows asymmetric enhancement of the left musculocutaneous nerve (arrow). Incidental note is made of bilateral subacromial/subdeltoid synovitis and left shoulder synovitis, which can contribute to shoulder pain but should not lead to shoulder weakness. D) Axial T2W FS MR image of the left upper arm redemonstrates thickening and oedema of the musculocutaneous nerve (arrowhead) and shows biceps brachii diffuse muscle oedema (arrow), corresponding to weakness with elbow flexion, which was the patient's most pronounced deficit on physical examination 

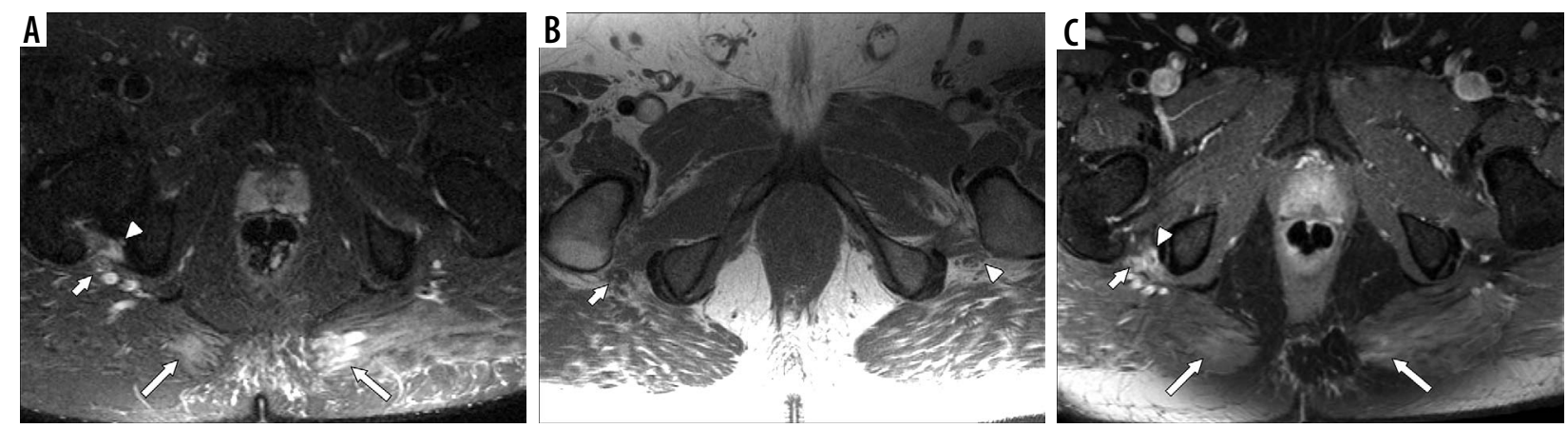

Figure 3. 1.5 T magnetic resonance neurography in a 37-year-old male with COVID-19-related acute respiratory distress syndrome and new-onset right distal lower extremity weakness and foot drop noted after extubation. A) Axial STIR large field-of-view magnetic resonance (MR) image of the lumbosacral plexus shows asymmetric right sciatic nerve thickening at the level of the ischial tuberosity (smaller arrow). There is adjacent focal quadratus femoris muscle oedema (arrowhead) and medial, bilateral gluteus maximus muscle oedema (longer arrows). These were of uncertain aetiology but could be related to pressure given the locations. B) Axial T1W NFS large field-of-view MR image of the lumbosacral plexus demonstrates asymmetric right sciatic nerve thickening and effacement of interfascicular fat (arrow) compared with the normal left side (arrowhead). C) Axial T1W FS post-contrast large field-of-view MR image of the lumbosacral plexus reveals sciatic nerve enhancement (shorter arrow) at the ischial tuberosity level that corresponds to oedema seen on the STIR sequence, and correlates to the site of the patient's suspected neuropathy based on clinical findings. The quadratus femoris muscle exhibits peripheral enhancement (arrowhead), which could represent focal myonecrosis. The medial gluteus maximus muscles are mildly enhancing as well (longer arrows)

Serum CK levels were elevated for 2 days, peaking 7 days after admission at $819 \mathrm{U} / \mathrm{l}$.

Electrodiagnostic studies could not be performed due to the patient's obesity. Because no focal radicular impingement was seen on lumbar spine MRI, lumbosacral plexus MRN was performed (Figure 3) and showed focal sciatic nerve oedema and enhancement at the level of the ischial tuberosity. The findings were thought to be due to compression and traction of the sciatic nerve at the ischial tuberosity, which occurred when the patient was in the prone position. There was adjacent quadratus femoris muscle oedema that was of uncertain aetiology. Post-contrast imaging of the quadratus femoris muscle showed an unusual peripheral enhancement, which suggested focal myonecrosis. In addition, the medial segments of the gluteus maximus muscles were also focally enhancing and oedematous, and the combination of muscle findings were thought to be positional and pressure-related, or possibly due to COVID-19 myositis.

\section{Discussion}

There are few descriptions of the imaging findings of peripheral neuropathy occurring in the extremities in patients with COVID-19 [4]. To our knowledge, this is the first case series describing the MRN findings seen in COVID-19 patients who develop symptomatic peripheral neuropathies in the extremities after the onset of severe respiratory symptoms. The causes of these conditions are not well known and may be multifactorial, including Guillain-Barré syndrome (GBS), critical-illness polyneuropathy (CIP)/critical-illness myopathy (CIM), positionrelated neuropathy, and toxic neuropathy.

GBS is an acute onset, rapidly progressive polyradiculopathy characterized by a symmetric, ascending, lower limb-predominant weakness that can also involve the facial muscle and muscles of respiration [5]. Males and older patients are more likely to develop this condition, and about $75 \%$ of cases are associated with antecedent respiratory or gastrointestinal infections, recently including COVID-19 [6]. GBS is an autoimmune process in which host antibodies attack nerve tissue, often due to a crossreactivity between an infecting agent and glycolipids in the peripheral nervous system, which can lead to demyelination or axonal loss [5].

Lumbar puncture may reveal a typical pattern of acellular cerebrospinal fluid with high protein concentration, and electrodiagnostic studies can show slowing of nerve conduction, delayed latency, and multifocal demyelinating polyneuropathy. However, the diagnosis is made clinically because lumbar puncture and electrodiagnostic studies may not begin to show characteristic abnormalities for 2-3 weeks $[7,8]$.

Imaging can be used to exclude other causes of symptoms, and postcontrast MRI of the lumbar spine can help support the diagnosis of GBS when there is a high index of clinical suspicion, particularly when lumbar puncture and electrophysiologic studies are equivocal, by showing surface thickening and enhancement of the conus medullaris and nerve roots of the cauda equina in cases of lower extremity involvement [9].

CIP and CIM are related complications of severe illness or trauma in critically ill patients, which often occur together. These entities classically present with a lower limb-predominant, diffuse, symmetric, flaccid muscle weakness, typically involving the hip and shoulder girdles, and can affect the phrenic nerve and respiratory muscles, which can exacerbate respiratory failure. Unlike GBS, they rarely involve the facial muscles, and females are more likely to develop CIP and CIM. These conditions usually present after 10-14 days in the ICU but can start to occur in as little as 72 hours in some patients. Patients with CIP have a worse prognosis for recovery [10]. CIP and CIM are probably the result of several pathways, includ- 
ing the following: microvascular alterations that lead to increased capillary permeability and increased cytokine release; metabolic derangements that activate proteolytic pathways; and electrical alterations that lead to diminished cell excitability. While electrodiagnostic studies can be helpful in establishing the diagnosis, and characteristic changes in electromyography (EMG) may precede functional impairment, some ICU patients may be unable to tolerate these studies [11].

MRI of the central nervous system (CNS) may help to exclude stroke or spinal infarct and limit the differential diagnosis. Peripherally, MRI of the proximal muscles has a nonspecific appearance, including symmetric distribution of diffuse muscle oedema and/or fatty infiltration, which can be seen with rhabdomyolysis or inflammatory myopathy. Muscle biopsy is considered the gold standard in detecting muscle pathology in early or mild cases, and muscle oedema seen on MRI can help direct tissue sampling [12].

Non-physiological positions performed over prolonged periods are a well-recognized cause of peripheral nerve injuries. Normally, traction of a peripheral nerve increases the target muscle activity and produces a nociceptive feedback response that causes the individual to change position and relieve the traction [13]. In patients who are obtunded or sedated, such as ventilated, perioperative, or critically ill patients, the feedback mechanism is disrupted, which may be exacerbated by neuromuscular relaxants, allowing individuals to maintain the nonphysiological positioning. Perioperative peripheral nerve injuries most commonly involve the upper extremity, and injuries of the ulnar nerve are most commonly reported, followed by those to the brachial plexus, and median and radial nerves [14].

As with other types of nerve injuries, the mechanism of position-related nerve injury is probably multifactorial, and most commonly a result of traction and compression. Stretching of a peripheral nerve by greater than 5-15\% of its resting length may predispose it to injury by increasing intraneural pressure and decreasing blood perfusion. This leads to subsequent ischaemia and endoneurial oedema. Nerve compression also increases intraneural and perineural pressure, leading to ischaemia, reduced nerve conduction, and localized axonal loss, and subsequent reperfusion can also lead to endoneurial oedema and demyelination [15].

Conditions producing microvascular disease, such as hypertension, smoking, diabetes mellitus, and general anaesthesia, are at greater risk for developing these injuries. Additionally, male patients, patients over 60 years of age, those with conditions such as hypotension, hypovolaemia and electrolyte disturbances, and orthopaedic and neurosurgical procedures that commonly place patients in vulnerable positions are also associated with position-related injuries [14].

ARDS frequently occurs with severe COVID-19 pneumonia, and prone positioning has long been recognized as an effective treatment to improve oxygenation in ARDS by reducing dorsal lung compression, increasing lung perfusion in these areas and improving the ventilation/ perfusion matching of the dorsal lung. Many institutions are performing prone-position ventilation more frequently on their critically ill, COVID-19-related ARDS patients [16]. However, proned patients may be susceptible to peripheral nerve injuries, especially in the upper extremity, because their shoulders may be posteriorly subluxated, hyperabducted, and externally rotated, and their elbows may be hyperflexed [15]. Recently, Malik et al. described their experience with 12 recovering patients with COVID-19 ARDS who sustained extremity peripheral nerve injuries at 21 sites. The authors found 11 of their 12 patients (92\%) had a history of at least one session of prone positioning during their initial treatment, and 16 of the 21 nerve injuries (76\%) occurred in the upper extremity, including to the ulnar nerve, radial nerve, and brachial plexus. A small number of injuries occurred in the lower extremities, and the authors reported 3 cases of sciatic neuropathy (14\%) [3].

As with other causes of peripheral neuropathy, the diagnosis of position-related nerve injuries usually requires a thorough history documenting a new-onset of symptoms sustained during surgery or during ICU treatment. Electrodiagnostic studies can be helpful to suggest the diagnosis [15]. Imaging of peripheral nerves in these patients has rarely been described and is not currently used to help diagnose these conditions. When performed, it has usually been done to exclude other causes for patient symptoms.

Although toxic neuropathies only represent $2-4 \%$ of peripheral neuropathies, they may be reversible if exposure to the offending material is stopped. Several substances, such as alcohol and a wide variety of medications, including chemotherapeutic agents, cardiovascular medications, CNS agents like nitrous oxide, antibiotic and antiviral medications, have been associated with peripheral neuropathies [17]. A recent report suggests remdesivir, an antiviral agent that was recently approved by the Food and Drug Administration to reduce the length of hospitalizations in patients with severe COVID-19-related respiratory syndromes, may also be associated with the development of peripheral neuropathies [18].

Many critically ill patients are exposed to numerous medications associated with toxic neuropathy to control severe infections, cardiovascular dysfunction, and oncologic complications, which can make it difficult to identify a contributing substance. However, even if a causative agent cannot be initially identified, some of them produce typical motor, sensorimotor, or sensory-predominant symptoms, which can help to narrow the list of potential offending agents. Electrodiagnostic studies can provide supportive evidence [17]. However, imaging is usually not critical to establishing the diagnosis of toxic neuropathy and is rarely performed. 
Imaging of peripheral nerves, such as MRI and ultrasound, is being increasingly used as an adjunct to the clinical examination and electrodiagnostic studies, to identify and localize segments of nerve pathology. Routine MRN has used a combination of high-resolution, T1-weighted (T1W), non-fat-suppressed (NFS) pulse sequences to provide edge detail in assessing the course and calibre of peripheral nerves as well as target muscle fatty infiltration, and heavily fluid-sensitive, fat-suppressed (FS) pulse sequences, such as T2-weighted (T2W) and short tau inversion recovery (STIR) sequences, which accentuates contrast in signal intensities between many pathological conditions and most normal background tissues. Newer, high-resolution 3D spin echo techniques have been performed to obtain thin-section imaging that can be reformatted in multiple planes, which can reduce volume averaging and improve the visualization of the peripheral nerves [19]. Peripheral nerves are often difficult to distinguish from adjacent blood vessels, and much ongoing research in MRN has focused on vascular suppression techniques. Although intravenous gadolinium contrast may help detect and characterize peripheral nerve tumours, inflammation, or perineural fibrosis, it may not improve identification of abnormal nerve segments for other indications [20]. However, intravenous contrast may be used to suppress vascular signal on 3D STIR spin echo sequences [19]. Finally, advanced MRN, including diffusion-weighted imaging and diffusion tensor imaging, are becoming more commonplace in characterizing the function of peripheral nerves, and have been used to characterize tumours and traumatic injuries.

Each of our cases demonstrated focal MRN abnormalities of one or more peripheral nerves, which corresponded to each patient's symptoms and physical examination findings. While abnormal nerve findings are visible on routine $2 \mathrm{D}$ spin echo sequences, these are better seen in our brachial plexus cases on thin-section, postcontrast $3 \mathrm{D}$ spin echo sequences in which there is robust vascular signal suppression. Furthermore, wide field-of-view, fat-suppressed, fluid-sensitive imaging and T1W NFS imaging that included the shoulder or hip girdles helped to identify patterns of muscle pathology, which can help identify abnormal peripheral nerves.

The precise cause of neuropathies in our cases has not been determined and may be due to several factors. However, there are common clinical and imaging features that may provide helpful insights into which COVID-19 patients are at particular risk for developing peripheral neuropathies in the extremities. All 3 of our patients were obese males who developed ARDS and required mechanical ventilation with at least one episode of prone positioning. The symptoms and imaging findings were unilateral or asymmetrical, which is less typical of systemic processes, such as GBS, CIP or toxic neuropathy, and all 3 patients noted some improvement or worsening of symptoms with changes in position. Furthermore, none of our patients noted rapid progression of their findings, which is characteristic of GBS. The MRN findings of CIP are not well established; however, patients with GBS commonly demonstrate more central abnormalities, such as surface enhancement of the cauda equina and nerve roots. In contradistinction, our patients demonstrated a lack of central enhancement, and focal oedema and enhancement of the involved, more distal peripheral nerves. Although our patients were exposed to several medications that have been associated with toxic neuropathies, their neuropathies did not resolve after discontinuing these medications, and the involved nerves have commonly been associated with position-related nerve injuries. Together, these findings suggest positioning, particularly placing the patient in the prone position during mechanical ventilation, may play a role in the development of extremity peripheral neuropathies in these patients.

\section{Conclusions}

Until now, the imaging findings in critically ill COVID-19 patients who have subsequently developed peripheral neuropathies in the extremities have rarely been reported. However, as the number of critically ill COVID-19 patients rises, we are starting to recognize these injuries more frequently and may be able to discern some emerging patterns that can help identify patients susceptible to these injuries. In particular, the use of prone positioning during mechanical ventilation, possibly in obese males, may be associated with subsequent unilateral or asymmetrical peripheral neuropathies that can be detected as segments of nerve oedema and enhancement on MRN corresponding to deficits reported by the patients and confirmed on physical examination. However, further work involving larger patient cohorts is necessary to determine the true impact of patient position and other factors on the development of peripheral neuropathies in these patients.

\section{Conflict of interest}

The authors report no conflict of interest.

\section{References}

1. "Home Page" Johns Hopkins Coronavirus Resource Center, Johns Hopkins University \& Medicine, November 15, 2020. Available at: https://coronavirus.jhu.edu.
2. Russell B, Moss C, Rigg A, et al. Anosmia and ageusia are emerging as symptoms in patients with COVID-19: what does the current evidence say? Ecancermedicalscience 2020; 14: ed98. 
3. Malik GR, Wolfe AR, Soriano R, et al. Injury-prone: peripheral nerve injuries associated with prone positioning for COVID-19related acute respiratory distress syndrome. Br J Anaesth 2020; 125: e478-e480.

4. Fernandez CE, Franz CK, Ko JH, et al. Imaging review of peripheral nerve injuries in patients with COVID-19. Radiology 2021; 298: E117-E130.

5. Walling AD, Dickson G. Guillain-Barre syndrome. Am Fam Physician 2013; 87: 191-197.

6. Dalakas MC. Guillain-Barre syndrome: the first documented COVID-19-triggered autoimmune neurologic disease: more to come with myositis in the offing. Neurol Neuroimmunol Neuroinflamm 2020; 7: e781.

7. van der Meché FG, van Doorn PA. Guillain-Barré syndrome and chronic inflammatory demyelinating polyneuropathy: immune mechanisms and update on current therapies. Ann Neurol 1995; 37 (Suppl 1): S14-S31.

8. Hadden RD, Cornblath DR, Hughes RA, et al. Electrophysiological classification of Guillain-Barré syndrome: clinical associations and outcome. Plasma Exchange/Sandoglobulin Guillain-Barré Syndrome Trial Group. Ann Neurol 1998; 44: 780-788.

9. Byun WM, Park WK, Park BH, et al. Guillain-Barre syndrome: MR imaging findings of the spine in eight patients. Radiology 1998; 208: 137-141.

10. Zhou Ch, Wu L, Ni F, et al. Critical illness polyneuropathy and myopathy: a systematic review. Neural Regen Res 2014; 9: 101-110.

11. Latronico N, Bolton CF. Critical illness polyneuropathy and myopathy: a major cause of muscle weakness and paralysis. Lancet Neurol 2011; 10: 931-941
12. Dinh A, Carlier R, Descatha A. Intensive critical illness myopathy and whole body MRI. Care Med 2016; 42: 587.

13. Coppieters MW, Van de Velde M, Stappaerts KH. Positioning in anesthesiology: toward a better understanding of stretch-induced perioperative neuropathies. Anesthesiology 2002; 97: 75-81.

14. Welch MB, Brummett CM, Welch TD, et al. Perioperative peripheral nerve injuries: a retrospective study of 380,680 cases during a 10-year period at a single institution. Anesthesiology 2009; 111: 490-497.

15. Kamel I, Barnette R. Positioning patients for spine surgery: avoiding uncommon position-related complications. World J Orthop 2014; 5: 425-443

16. Golestani-Eraghi M, Mahmoodpoor A. Early application of prone position for management of Covid-19 patients. J Clin Anesth 2020; 66: 109917.

17. Toledano M. Toxin-induced neuropathies. Neurol Clin 2020; 38: 749-763.

18. Jorgensen SCJ, Kebriaei R, Dresser LD. Remdesivir: review of pharmacology, pre-clinical data, and emerging clinical experience for COVID-19. Pharmacotherapy 2020; 40: 659-671.

19. Zhang Y, Kong X, Zhao Q, et al. Enhanced MR neurography of the lumbosacral plexus with robust vascular suppression and improved delineation of its small branches. Eur J Radiol 2020; 129: 109128.

20. Harrell AD, Johnson D, Samet J, et al. With or without? A retrospective analysis of intravenous contrast utility in magnetic resonance neurography. Skeletal Radiol 2020; 49: 577-584. 